\title{
Mechanics of diagnostic machine on electrical transmission lines conductors
}

\author{
Mohammad Reza Bahrami ${ }^{1, *}$ \\ ${ }^{1}$ Peter the Great St.-Petersburg polytechnic university, St.-Petersburg, Russian Federation
}

\begin{abstract}
The article deals with the dynamics of wire transmission line while the robot-inspector is moving on it. To ensure proper and safe operation of the machine, mathematical modeling based on the Lagrangian mechanics has been conducted. The wire is considered as a stretched string with additional bending stiffness, and the robot-inspector as moving mass and pendulum. As the result, saw-tooth oscillations have been observed in the vertical plane and they cause parametric oscillations in the perpendicular plane. Advanced method of mathematical modeling of inspection robot motion on conductors allows us to choose the design parameters and the law of motion, in order to prevent accidents and ensure the safety of personnel.
\end{abstract}

\section{Introduction}

Nowadays electrical transmission lines play significant roles in human life. The electricity power transmits through transmission lines from the power plants to the cities and industrial centers. Consequently, if any damage and disruption of these transmission line human life and industries might face problems. Inspection and maintenance of electrical transmission lines are necessary in order to prevent further damages in the mentioned areas.

On the other hand, inspection of electrical transmission lines performed by human forces would be faced with danger, but by improvement of technology, robots have been used as reliable machines instead of human forces in potentially dangerous environments such as hot-lines. Currently, power companies mainly use robots for inspection tasks by considering the fact that repair works are often complicated to be accomplished by a robot, especially on the electrical lines.

Robotic inspection is the utilization of intelligent or remotely controlled machines that combine technologies such as imaging, sensing to inspect the condition of transmission line components. The mission is to reduce or eliminate human exposure to potentially dangerous environments while inspecting the electrical transmission lines in order to decrease the cost of repair.

Inspection of electrical transmission lines attracts the attention of many researchers, and in some countries, to check the status of the overhead electrical transmission lines special automatic diagnostic machines (ADM) (inspection robots) [1] had created. A novel electrical transmission line inspection robot which was presented in [2] is shown in Fig.1. It

${ }^{*}$ Corresponding author: mr.bahrami@inbox.ru 
includes a motorized drive wheel, with a gripper and a central main box. The machine can pass through different obstacles on electrical transmission lines (dampers, clamps, warning balls).

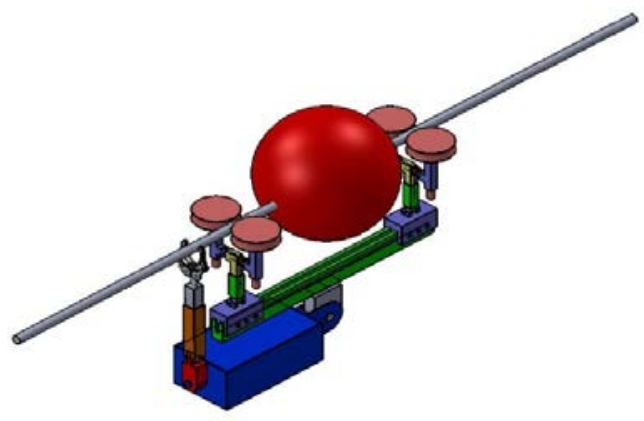

Fig. 1. Schematic of inspection robot.

However, the operation of such devices may be compromised because of the high ductility of the conductor [3-9]. Even with the slow movement of ADM, it is possible that the dangerous vibrations appear which are leading to failure or breakage [10-14]. Thus mathematical modeling of motion of ADM on the conductor is necessary at the design stage in order to select the appropriate options for reducing the cost of the experiment and to protect personnel.

The purpose of this article is the development of modeling technique and its application. The technique involves finding the dynamics equations of ADM on conductors and solves them by means of computer mathematical software. The equations are derived based on mechanics of Lagrange for solid and deformable bodies [15-16], and by means of computational tools, Mathcad [17].

\section{String with a moving mass on it}

In the first section, the dynamics of the wire with a moving mass is considered (Fig. 2). The equation of oscillations of wire in the vertical plane has the following form

$$
E J u^{\prime \prime \prime}+T u^{\prime \prime}=p(x, t)-\rho \ddot{u}
$$

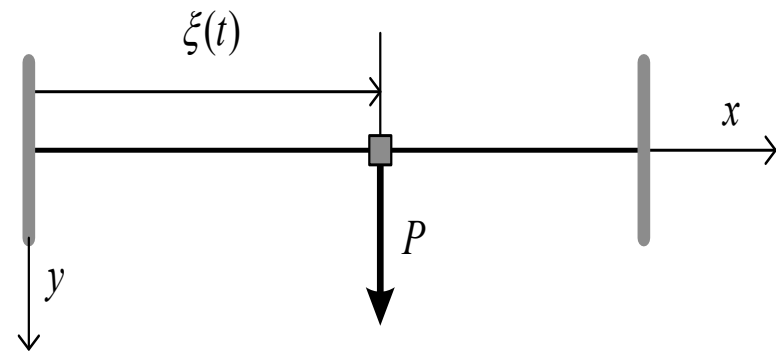

Fig. 2. Wire with a movable mass on it.

Here $u(x, t)$ is the deflection, $E J$ is the flexural rigidity, $T$ is the tension force, $p$ is the distributed load, $\rho$ is the mass per unit length. The boundary conditions at the ends of the hinge-support: $u=0, u^{\prime \prime}=0$. Load $p$ contains a concentrated force of inertia of the moving mass, which greatly complicates the solution of equation (1). 
However, it is possible to construct an approximate solution by using Lagrange equations. Coordinates and velocity of the center of mass are:

$$
\begin{aligned}
& x=\xi(t), y=u(\xi(t), t)=U(t) . \\
& \Rightarrow \dot{x}=\dot{\xi}, \dot{y}=u^{\prime} \dot{\xi}+\dot{u}
\end{aligned}
$$

Generalized coordinates of the system $q_{i}(i=1 \ldots n)$ are introduced by deflection approximation as:

$$
\begin{aligned}
& u(x, t)=\sum_{i=1}^{n} q_{i}(t) \varphi_{i}(x)=q(t)^{T} \varphi(x), \\
& \varphi_{i}=\sin i \pi x / l .
\end{aligned}
$$

The kinetic energy is determined by (twice, with matrix notation):

$$
\begin{aligned}
& 2 K=m\left(\dot{x}^{2}+\dot{y}^{2}\right)+\rho \int_{0}^{l} \dot{u}^{2} d x= \\
& =m \dot{\xi}^{2}+\dot{q}^{T}[M+\mu(\xi)] \dot{q}+2 \dot{\xi} q^{T} \eta(\xi) \dot{q}+\dot{\xi}^{2} q^{T} \sigma(\xi) q ; \\
& M=\int_{0}^{l} \rho \varphi \varphi^{T} d x \\
& \mu=m \varphi(\xi) \varphi^{T}(\xi), \\
& \sigma=m \varphi^{\prime}(\xi) \varphi^{\prime T}(\xi), \\
& \eta=m \varphi^{\prime}(\xi) \varphi^{T}(\xi) .
\end{aligned}
$$

Next, the potential energy is determined by

$$
\begin{aligned}
& \Pi=\frac{1}{2} \int_{0}^{l}\left(T u^{\prime 2}-E J u^{\prime \prime 2}\right) d x-P u(\xi, t)=\ldots \\
& \ldots=\frac{1}{2} q^{T} C q-q^{T} F(\xi), \\
& C=\int_{0}^{l}\left(T \varphi^{\prime} \varphi^{\prime T}+E J \varphi^{\prime \prime} \varphi^{\prime \prime T}\right) d x, \\
& F=P \varphi(\xi) .
\end{aligned}
$$

Now, Lagrange equation can be formed as:

$$
\begin{aligned}
& \left(\frac{\partial K}{\partial \dot{q}}\right)^{\bullet}-\frac{\partial K}{\partial q}=-\frac{\partial \Pi}{\partial q} \Rightarrow \\
& \Rightarrow \underline{(M+\mu) \ddot{q}+\left[C+\dot{\xi}^{2} \kappa(\xi)\right] q+2 \dot{\xi} \eta^{T}(\xi) \dot{q}=F}
\end{aligned},
$$

in which $\kappa=m \varphi \varphi^{\prime \prime T}$.

Underlined part in (6) is transformed to the canonical form of the ODE system for the subsequent numerical integration: 


$$
\left\{\begin{array}{l}
\dot{q}=w \\
\dot{w}=(M+\mu)^{-1}\left[F-\left(C+\dot{\xi}^{2} \kappa\right) q+2 \dot{\xi} \eta^{T} w\right]
\end{array} .\right.
$$

Numerical solution (when initial conditions equal to zero) has been done by Mathcad. The system (7) is somehow rigid, so the selected method in Mathcad is Radau. Fig. 3 shows the results of calculation of the deflection $U(t)=u(v t, t)$ and acceleration $a(t)=\ddot{U}(t)$ with a constant speed $\dot{\xi}=v=2 \mathrm{~m} / \mathrm{s}$.Values of the parameter are: $E J=2.15 \times 10^{4}, T=10^{4}$, $\rho=5.3, m=100, l=200$ (units according to SI system). The number of members in the approximation is $n=17$.
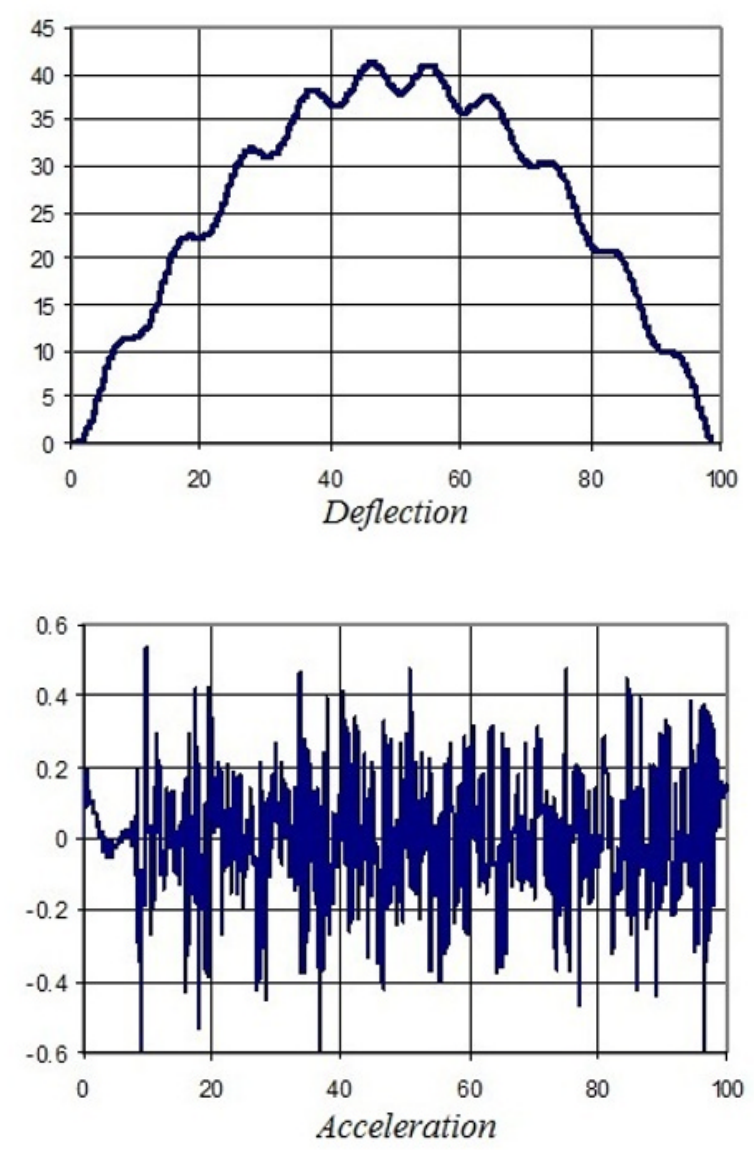

Fig. 3. Deflection (top) and acceleration (bottom) of the conductor.

Surprisingly, the saw-tooth oscillations in the wires graph in the vertical plane appear when the motion of a concentrated mass is steady. These vibrations cause inertial load on the construction of the robot with overload $a(t)$.

\section{Pendulum with a movable suspension base}

The consequence of these saw-tooth oscillations can also be parametric oscillations of the robot as a pendulum with movable suspension base (Fig.4). 


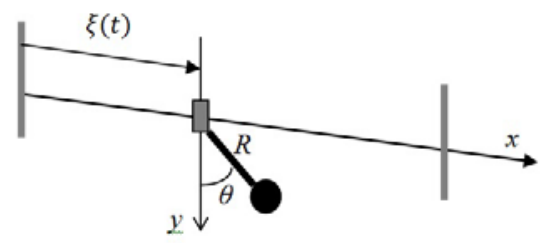

Fig. 4. Pendulum with a movable suspension base.

In this case equation of a pendulum has been used:

$$
\ddot{I}+(g-a(t)) R \theta=0 .
$$

Here $\theta(t)$ is the angle of deflection of the pendulum from the vertical axis, $I$ is the moment of inertia about the axis of suspension, $R$ is the distance from the center of gravity to the suspension point. It is known that with the periodic dependence $a(t)$, it is possible that parametric resonance appears which are more dangerous than forced oscillations cases. Parametric excitation differs from forcing since the action appears as a time-varying modification on a system parameter.

In our case, it is not the classical parametric resonance, since there is no periodic excitation, however dangerous oscillations with increasing amplitude is possible. Study of this character can be done by mathematical modeling; by setting $\theta(0)=0.1, \dot{\theta}(0)=0$ and $a(t)$ as shown in Fig.3, numerical solution of (8) been obtained. Fig.5. shows the result.

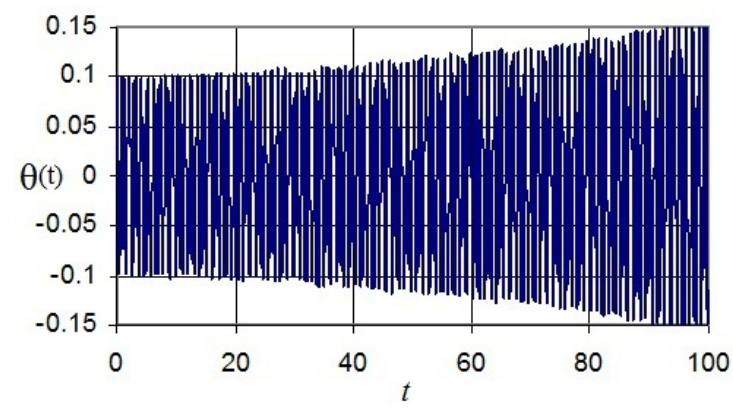

Fig. 5. Parametric oscillations of the pendulum.

It can be seen that the growth of fluctuations, limited only by the time of the process, here $l / v=100 \mathrm{~s}$. The pendulum parameters were chosen specifically so that the vibrations grew. This example shows the possibility of a kind of parametric resonance.

\section{Conclusions}

Mathematical modeling and calculation of electrical transmission line oscillations as the result of inspection robot movement on it has been studied. Identified fluctuations as the results are: dangerous saw-tooth fluctuations in the vertical plane and they cause parametric fluctuations. Multivariate calculations have shown that the parametric resonance can be avoided. The procedure can be summarized as taking into account the output wires of the plane defined by the engine power and changes in the structure of the machine during 
operation and avoiding obstacles. Practical results of the calculations can be used in the selection of design parameters and speed.

\section{References}

1. K. Toussaint, N. Pouliot, S. Montambault, Transmission line maintenance robots capable of crossing obstacles: state-of-the-art review and challenges ahead, J. of Field Rob., 26(5), pp. 477-499 (2009).

2. M.R. Bahrami, A novel design of electrical transmission line inspection machine, Lect. Notes in Mech. Eng.: Adv. in Mech. Eng., ISBN: 978-3-319-29578-7, pp. 67-73 (2016).

3. S. Aoshima, T. Tsujimura, T.A. Yabuta, Wire mobile robot with multi-unit structure, IEEE/RSJ Int.W. on Intel. Rob. and Sys., pp. 414-421 (1989).

4. J. Sawada, K. Kusumoto, T. Munakata, Y. Maikawa, Y.A. Ishikawa, Mobile robot for inspection of power transmission lines, IEEE Trans. on Power Del., 6(1), pp. 309-315 (1991).

5. M. Higuchi, Y. Maeda, S. Tsutani, S. Hagihara, Development of a Mobile Inspection Robot for Power Transmission Lines, J. of Robotic Soc. of Japan, 9(4), pp. 57-63 (1991).

6. T. Tsujimura, T. Morimitsu, Dynamics of mobile legs suspended from wire, Rob. and Aut. Sys., 20(1), pp. 85-98 (1997).

7. S. Montambault, N. Pouliot, Design and validation of a mobile robot for power line inspection and maintenance, 6th Int. Conf. on Field and Ser. Rob. (FSR), pp. 1-10 (2007).

8. Zhou, Fengyu, Xiao, Hairong, Wu, Aiguo, Control strategy and implementation of an inspection robot for $110 \mathrm{kv}$ power transmission lines, 6th W. C. on Intel. Control, China, (2006).

9. P. Debenest, M. Guarnieri, K. Takita, and others, Robot for Inspection of Transmission Lines, IEEE Int. Conf. on Rob. and Aut., Pasadena (2008).

10. R.Sh. Gimadiev, F.F. Dinmukhametov, N.R. Galimullin, Calculation of the spatial dynamics of power lines under the combined effect of wind and weight load, J. Problems of energy, 3-4, pp. 28-37 (2010).

11. V. V. Eliseev, M. R. Bahrami, Diagnostic machine on power transmission lines: configuration and mechanical challenges, St. Petersburg Poly. Uni. J., 1 (214), pp. 200-207 (2015).

12. V.V. Eliseev, M.R. Bahrami, Dynamic of electrical transmission lines with inspection robot on it, Proc. of the uni. Instrum., 3 (58), pp. 229-235 (2015).

13. V.V. Eliseev, M.R. Bahrami, Strength suspension of inspector robot on the electrical transmission line wire, J. of Mech. Eng., 6, pp.19-22 (2016).

14. V. V. Eliseev, M. R. Bahrami, Dynamic of electrical transmission lines with inspection robot on it under influence of limited power of robot engine, Theory of Mech. and Mach. J., St. Petersburg, 4, pp. 6-11 (2015).

15. V.V. Eliseev, Mechanics of Deformation of Rigid Body. (Polytech. Uni. Pub., St. Petersburg, Russia, 2006).

16. V.V. Eliseev, Y. Vetyukov, Effects of deformation in the dynamics of belt drive. Acta Mechanica, 223, pp. 1657 - 1667 (2012).

17. D.V. Kiryanov, Mathcad 14, p. 704 (St. Petersburg: BHV-Petersburg, 2007). 